\title{
Sinterização de cerâmicas à base de PZT em forno de microondas
}

\section{(Microwave synthesis of PZT based ceramics)}

\author{
M. V. Gelfuso, F. Teixeira Neto, D. Thomazini \\ Laboratório de Materiais e Instrumentação Eletrônica - Universidade de Fortaleza \\ Av. Washington Soares 1321, Fortaleza, CE \\ virginia@unifor.br
}

\begin{abstract}
Resumo
Cerâmicas de titanato e zirconato de chumbo dopadas com neodímio foram sinterizadas em um forno de microondas de uso doméstico modificado. Neste tipo de aquecimento, a energia eletromagnética é transferida diretamente para o material, sendo convertida em calor pelo material cerâmico, reduzindo o tempo e energia durante o processamento. Desta forma, foram produzidos corpos cerâmicos com tempo de sinterização que variou até $30 \mathrm{~min}$, a 1100 e $1200{ }^{\circ} \mathrm{C}$. As fases foram investigadas por meio de difratometria de raios X. Corpos cerâmicos com densidade de $95 \%$ da teórica foram obtidos com tempo de sinterização de 10 min em $1100{ }^{\circ} \mathrm{C}$, com uma taxa de aquecimento de $400{ }^{\circ} \mathrm{C} / \mathrm{min}$. Para as cerâmicas com densidade superior a $90 \%$ da teórica foram medidos os valores de constante dielétrica e fator de perda.
\end{abstract}

Palavras-chave: sinterização, microondas, PZT, propriedades dielétricas.

\section{Abstract}

Ferroelectric ceramics based on lead zirconate titanate doped with neodymium were sintered using a modified microwave oven. In this process the energy is transferred directly to the material through the interaction between molecules of the material with the electromagnetic field. The microwave sintering process has an advantage to reduce the time and save energy. This type of process involves energy conversion, opposing to heat transfer. Ceramic parts were produced by thermal treatments at $1100^{\circ} \mathrm{C}$ and $1200^{\circ} \mathrm{C}$ up to $30 \mathrm{~min}$. The formed crystalline phases were investigated by X-ray diffraction. Ceramic parts with densities above $95 \%$ of the theoretical density were produced in 10 min by heating at $1100^{\circ} \mathrm{C}$, with heating rate of $400^{\circ} \mathrm{C} / \mathrm{min}$. Values of dielectric constant and loss factor of ceramics with density above 90\%T.D. were determined.

Keywords: microwave sintering, PZT, dielectric properties.

\section{INTRODUÇÃO}

Cerâmicas com estruturas perovskitas a base de chumbo são conhecidas por serem um dos principais materiais ferroelétricos utilizados comercialmente, como capacitores multicamadas, transdutores piezoelétricos, detetores piroelétricos e sensores ópticos. As propriedades destas cerâmicas são fortemente influenciadas pela densidade e microestrutura do material, as quais dependem do processo de síntese do pó e processamento [1-4]. É conhecido que no processo convencional de sinterização destas cerâmicas a temperatura de queima é $1200^{\circ} \mathrm{C}$, o que para longos tempos de sinterização provoca alta volatilização de $\mathrm{PbO}$. Essa perda de massa não só ocasiona a deterioração das propriedades elétricas, dielétricas e piezoelétricas das cerâmicas como também aumenta o custo de processamento e causa danos ao meio ambiente. Contudo, se a taxa de aquecimento é elevada, a volatilização do $\mathrm{PbO}$ pode ser suprimida e o refinamento dos grãos pode ser melhorado [5-7]. Neste sentido a sinterização em fornos de microondas vem sendo proposta como uma alternativa para sinterizar cerâmicas ferroelétricas a base de perovskitas de chumbo a taxas de aquecimentos elevadas em intervalos de tempo reduzidos [8-10]. A sinterização das cerâmicas através de microondas proporciona estas condições de tratamento térmico e apresenta-se como um método alternativo para sinterizar o material, pois envolve conversão de energia, em lugar de transferência de calor [11]. A utilização de microondas promove maior homogeneidade estrutural e menor tamanho médio de grão, em comparação com a sinterização em forno resistivo, além de apresentar como vantagem a redução do tempo e a economia de energia $[12,13]$.

$\mathrm{O}$ efeito da adição de óxidos de elementos terras-raras nas cerâmicas de PZT tem sido extensivamente estudado, se mostrando eficiente no aumento da constante dielétrica, parâmetros eletromecânicos e redução no fator de qualidade mecânica [14]. Porém pouco se sabe a respeito do comportamento destes compostos quando sinterizados em microondas. Assim, neste trabalho foi estudado o processo de sinterização de cerâmicas de $\mathrm{PZT}$ próximas ao contorno de fase morfotrópico com adição de $\mathrm{Nd}_{2} \mathrm{O}_{3}$ em forno de microondas e foram analisadas as propriedades físicas, dielétricas e aspectos microestruturais destas cerâmicas. 


\section{PROCEDIMENTO EXPERIMENTAL}

Pós com composição nominal $\mathrm{Pb}\left(\mathrm{Zr}_{0,53} \mathrm{Ti}_{0,47}\right) \mathrm{O}_{3}+1$ peso $\% \mathrm{Nd}_{2} \mathrm{O}_{3}$ (PZT:Nd) foram preparados através do método de mistura convencional de óxidos. Os materiais precursores foram pós de $\mathrm{PbO}$ litargírio (99,9+\% da Litargo), $\mathrm{TiO}_{2}$ tetragonal (99,8+\% da Vetec) e $\mathrm{ZrO}_{2}$ monoclínico $(99,6+\%$ da Vetec). Os óxidos foram misturados em moinho de bolas durante $3,5 \mathrm{~h}$, e calcinados a $850{ }^{\circ} \mathrm{C}$ durante $3,5 \mathrm{~h}$ ao ar. Os pós de PZT:Nd foram moídos em frasco de polietileno contendo água destilada, defloculante e esferas de zircônia de 1,0 $\mathrm{mm}$ de diâmetro obedecendo a razão em volume do frasco de moagem de $50 \%$ com cilindros de zircônia estabilizada e $25 \%$ de pó. A distribuição dos tamanhos de partícula dos pós foi obtida após dois minutos de agitação ultra-sônica para quebra dos agregados, através de um analisador de distribuição de tamanho de partículas Horiba modelo Capa-700.

A temperatura de calcinação de $850{ }^{\circ} \mathrm{C}$ foi determinada após a análise térmica diferencial (ATD) realizada em um equipamento Netzsch STA-409. Os pós foram conformados no formato de disco de 20,0 $\mathrm{mm}$ de diâmetro e 1,0 $\mathrm{mm}$ de espessura através de prensagem uniaxial a $30 \mathrm{MPa}$. O valor da densidade a verde das cerâmicas foi de aproximadamente $50 \%$ da densidade teórica.

As cerâmicas foram sinterizadas no intervalo de 0 até 30 minutos a 1100 ou $1200{ }^{\circ} \mathrm{C}$ ao ar, em forno de microondas doméstico Panasonic, apresentando câmara de $350 \mathrm{~mm}$ x $230 \mathrm{~mm}$ e freqüência $2,45 \mathrm{GHz}$ (Fig. 1) com uma potência nominal de $1450 \mathrm{~W}$. A temperatura interna do forno foi

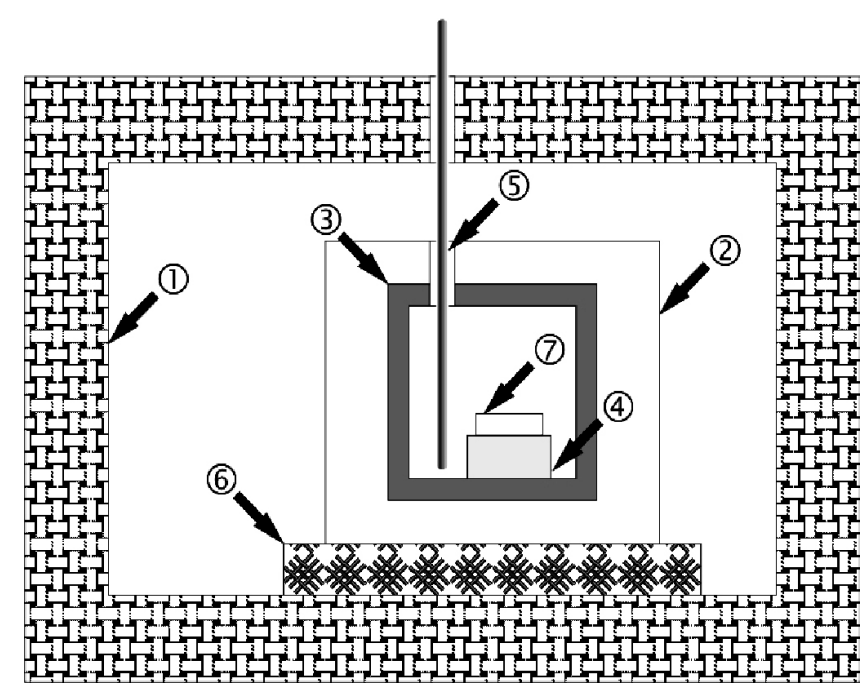

Figura 1: Representação esquemática do sistema de aquecimento por microondas. 1- Câmara do forno, 2- Isolante térmico de fibra de alumina, 3- Absorvedor de microonda ( $\mathrm{SiC})$, 4- Base de alumina, 5- Termopar, 6- Isolante térmico de zircônia, 7- Amostra de PZT.

[Figure 1: Schematic representation of the microwave heating system. 1- Oven chamber, 2- Fibrous alumina thermal insulator, 3- SiC microwave absorber, 4- Alumina base, 5- Thermocouple, 6- Zirconia plate, thermal insulator, 7-PZT specimen.] medida com um termopar tipo $\mathrm{K}$ blindado em aço inox para evitar sua interação com a radiação eletromagnética. Neste estudo o susceptor com formato de anel foi alocado em torno da amostra, proporcionando a homogeneização da irradiação térmica na cerâmica. O susceptor de $\mathrm{SiC}$ promove o aquecimento inicial dos corpos de PZT necessário para que a própria amostra mantenha seu auto-aquecimento por dissipação dielétrica [15].

As densidades das amostras sinterizadas foram obtidas por meio do método de Arquimedes. As superfícies das amostras foram analisadas com o auxílio de um microscópio eletrônico de varredura (Zeiss DSM-940A). Eletrodos de prata foram depositados sobre as superfícies planas e paralelas das amostras para as medidas de capacitância e fator de dissipação dielétrica. As medidas foram realizadas com um capacímetro Minipa MX 1001. Os valores de constante dielétrica $(\varepsilon)$ e fator de perda dielétrica $(\operatorname{tg} \delta)$ foram medidos a $1 \mathrm{kHz}$ em temperatura ambiente.

\section{RESULTADOS E DISCUSSÃO}

A Fig. 2 mostra as curvas de análise térmica diferencial (ATD) e análise termogravimétrica (ATG) da mistura de óxidos precursores para composição PZT:Nd. Observa-se uma reação endotérmica a $300{ }^{\circ} \mathrm{C}$ devido a perda de água ligada e de orgânicos como vestígios de frasco de moagem e defloculante. Pode ser observada uma reação exotérmica iniciada a $850{ }^{\circ} \mathrm{C}$ típica de curvas de ATD realizadas em compostos de PZT cuja formação ocorre nesta faixa de temperatura [16]. A presença da fase PZT pôde ser confirmada através de análise de difração de raios X (Fig 5). $\mathrm{Na}$ Para temperaturas acima de $1000{ }^{\circ} \mathrm{C}$ pode ser visto uma crescente perda de massa devido à volatilização do $\mathrm{PbO}$.

O efeito do tempo de sinterização na densidade das amostras está ilustrado na Fig. 3. O processo de sinterização é iniciado com 2 min de queima devido ao fato da interação

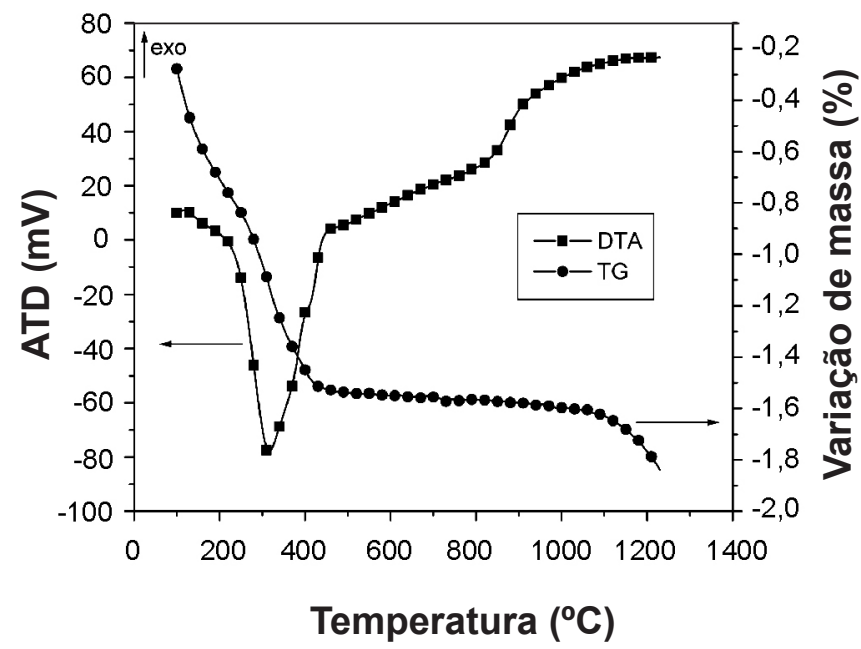

Figura 2: Análises termogravimétrica e térmica diferencial do pó de PZT-Nd.

[Figure 2: DTA and TGA of PZT:Nd powder.] 


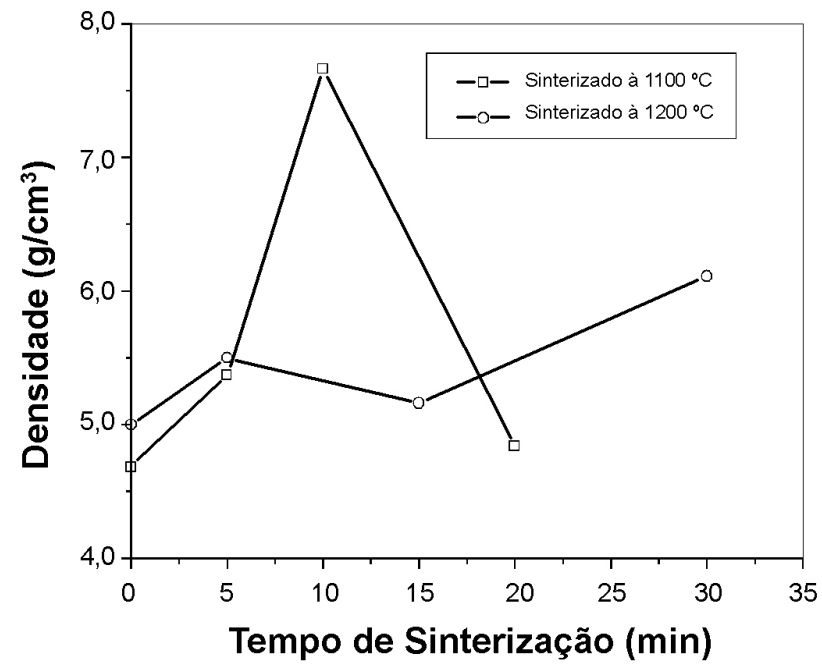

Figura 3: Curvas de densificação para cerâmicas de PZT:Nd sinterizadas a 1100 e $1200^{\circ} \mathrm{C}$ até $30 \mathrm{~min}$.

[Figure 3: Densification curves for PZT:Nd ceramics sintered at 1100 and $1200^{\circ} \mathrm{C}$ up to 30 minutes.]

eletromagnética com o material cerâmico promover um aquecimento volumétrico através da dissipação dielétrica, o qual intensifica o processo de sinterização em poucos minutos. Estudos realizados revelaram que a energia de ativação necessária para sinterizar corpos de alumina em forno de microondas é um terço menor que aquela observada no método convencional [17]. Assim, as cerâmicas sinterizadas em microondas são densificadas em menos tempo. O maior valor de densidade foi obtido para cerâmicas sinterizadas em $1100{ }^{\circ} \mathrm{C}$ durante $10 \mathrm{~min}$, sendo obtido um valor de $7,65 \mathrm{~g} / \mathrm{cm}^{3}$ ( 95\% da densidade teórica). As cerâmicas sinterizadas em temperatura acima de $1200{ }^{\circ} \mathrm{C}$ apresentaram

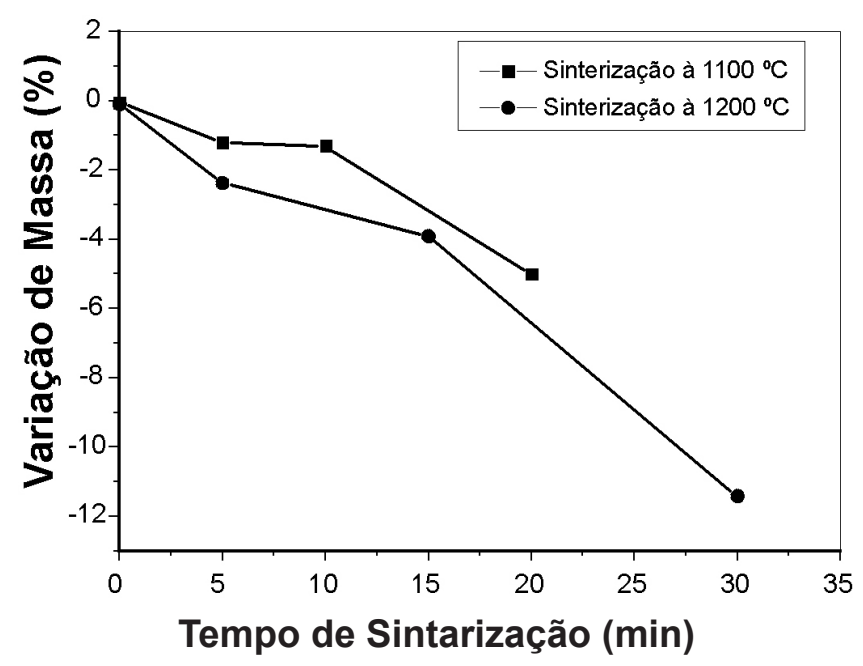

Figura 4: Perda de massa em função do tempo de patamar para cerâmicas queimadas a $1100^{\circ} \mathrm{C}$ e $1200^{\circ} \mathrm{C}$.

[Figure 4: Mass loss as a function of soaking time for ceramics fired at $1100^{\circ} \mathrm{C}$ and $1200^{\circ} \mathrm{C}$.] início do processo de fusão, confirmado por meio de estudos anteriores, mostrando que o óxido de neodímio forma uma fase líquida com o $\mathrm{PbO}$ nesta temperatura [3].

Para amostras sinterizadas em tempos maiores que de 15 min, altos valores de retração geométrica foram observados porém a perda de massa foi favorecida, reduzindo os valores de densidade das cerâmicas. A Fig. 4 ilustra a variação de massa em função do tempo de sinterização das cerâmicas sinterizadas a 1100 e a $1200{ }^{\circ} \mathrm{C}$. A maior densificação na temperatura de $1200{ }^{\circ} \mathrm{C}$ foi verificada para a cerâmica sinterizada durante $30 \mathrm{~min}$ (75\% da densidade teórica), porém a perda de massa foi de aproximadamente 1,2\% a qual prejudicou o processo de densificação [3].

A Fig. 5 mostra o difratograma de raios $\mathrm{X}$ para o pó calcinado e amostra sinterizada de PZT:Nd a $1100{ }^{\circ} \mathrm{C}$ durante 10 min. A similaridade entre os padrões de difração sugere a ausência de fases diferentes do PZT devido ao processamento por microondas.

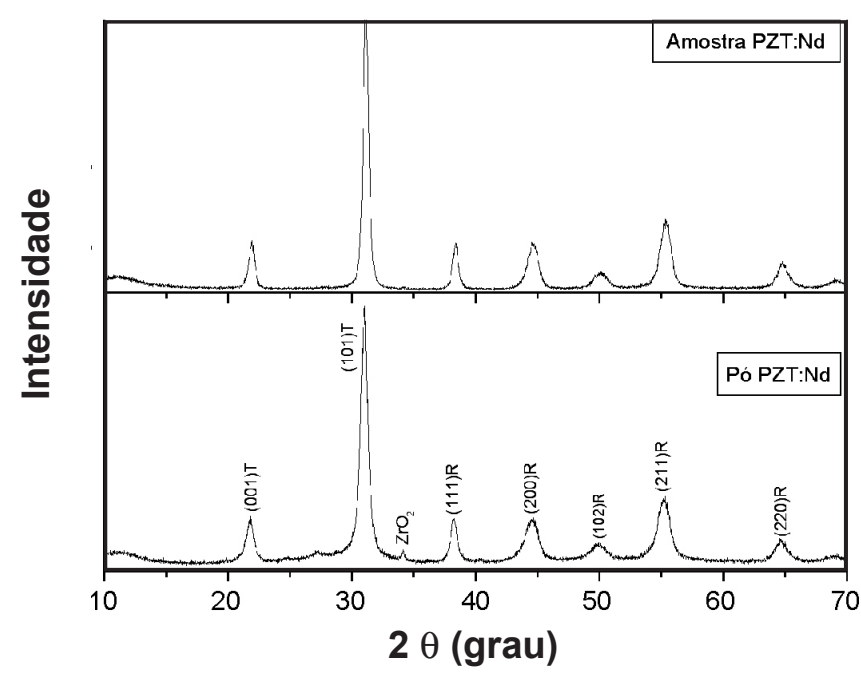

Figura 5: Difratogramas de raios $\mathrm{X}$ de PZT:Nd: Pó calcinado $\left(850{ }^{\circ} \mathrm{C} / 3,5 \mathrm{~h}\right)$ e cerâmica queimada a $1100{ }^{\circ} \mathrm{C}$ durante $10 \mathrm{~min}$ em forno de microondas.

[Figure 5: XRD patterns of PZT:Nd: Calcined powder $\left(850^{\circ} \mathrm{C} / 3.5 \mathrm{~h}\right)$ and ceramic fired at $1100{ }^{\circ} \mathrm{C}$ during $10 \mathrm{~min}$ by microwave sintering.]

A Fig. 6A mostra a superfície de uma cerâmica sinterizada a $1100{ }^{\circ} \mathrm{C}$ durante $10 \mathrm{~min}$. $\mathrm{O}$ valor obtido da constante dielétrica para esta amostra foi de 2426 e o valor do fator de perda dielétrico foi de aproximadamente 3,0\%. Este é um alto valor de constante dielétrica quando comparado ao de $\varepsilon=1200$ para cerâmicas obtidas pelo processo convencional de sinterização [3], podendo ser atribuído ao tamanho reduzido dos grãos que promove o ancoramento dos dipolos elétricos [6]. As Figs. 6B e 6C mostram as micrografias das cerâmicas sinterizadas durante 15 e 30 min a $1200{ }^{\circ} \mathrm{C}$. Pode ser observado que estas amostras apresentaram início do processo de fusão, o que impossibilitou a obtenção de resultados de medidas dielétricas confiáveis. Para as amostras sinterizadas sem tempo de patamar, ou seja, 

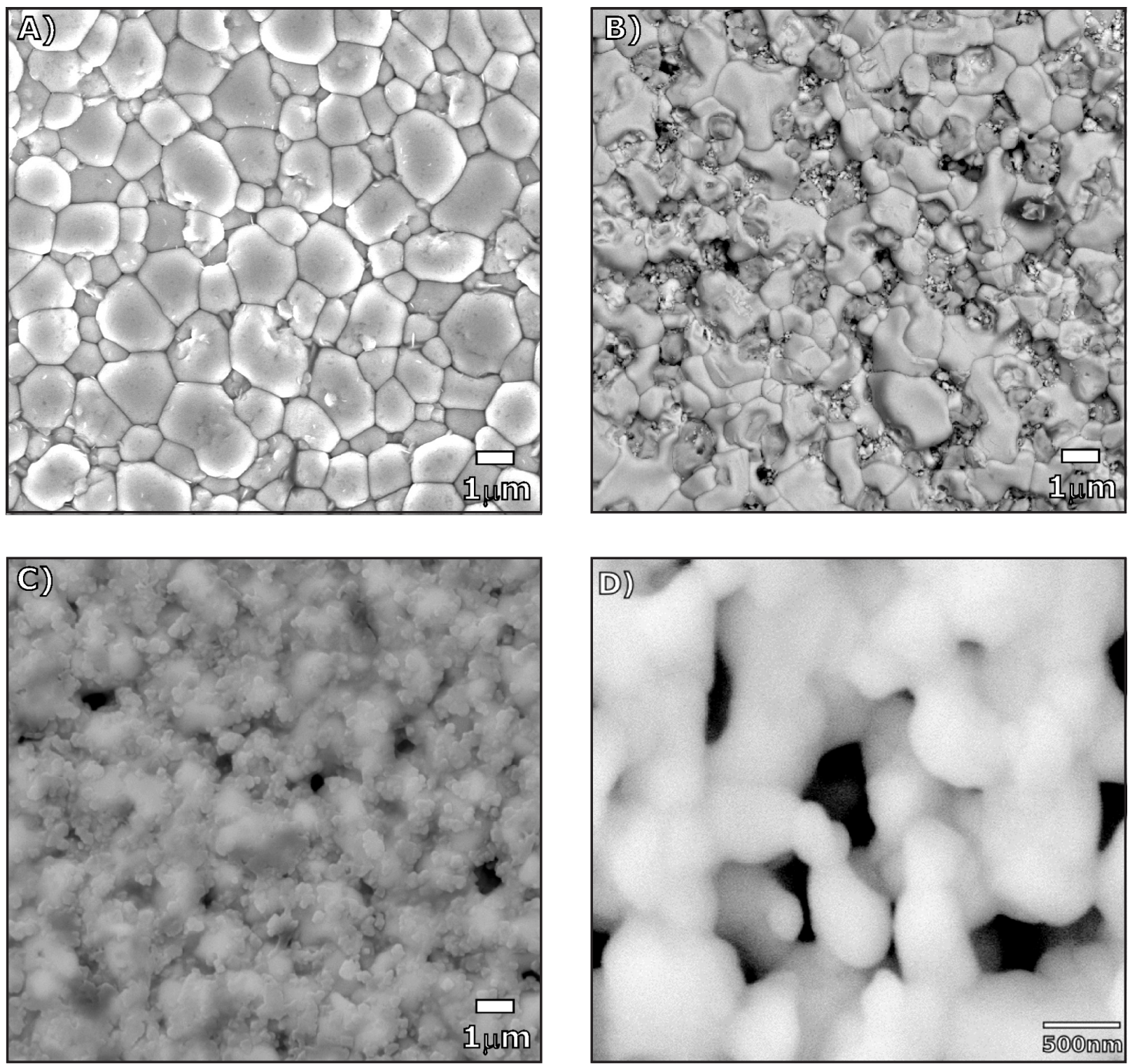

Figura 6: Micrografias obtidas por microscopia eletrônica de varredura de cerâmicas de PZT sinterizadas. A) $1100{ }^{\circ} \mathrm{C}$ durante 10 min e $1200{ }^{\circ} \mathrm{C}$ durante: B) $15 \mathrm{~min}$ e C) $30 \mathrm{~min}$; e D) $1100^{\circ} \mathrm{C}$ sem patamar.

[Figure 6: SEM micrographs of PZT sintered ceramics. A) $1100^{\circ} \mathrm{C}$ during 10 min and $1200^{\circ} \mathrm{C}$ during: B) 15 min and C) 30 min; and D) $1100^{\circ} \mathrm{C}$ without soaking time.]

amostras que foram aquecidas e imediatamente resfriadas ao atingir a temperatura proposta, $1100{ }^{\circ} \mathrm{C}$ e $1200{ }^{\circ} \mathrm{C}$, pôde ser observado que o processo de sinterização foi iniciado devido a formação de empescoçamento entre os grãos (Fig. 6D), porém o processo de densificação não foi finalizado devido a presença de poros abertos na microestrutura. O tamanho médio dos grãos para a cerâmica sinterizada a $1100{ }^{\circ} \mathrm{C}$ durante $10 \mathrm{~min}$ foi de aproximadamente 1,0 $\mu \mathrm{m}$. Em estudos anteriores [3] o tamanho médio dos grãos para cerâmicas sinterizadas através do processo convencional foi $2,0 \mu \mathrm{m}$, sugerindo que o processo de densificação é dominante em relação ao de crescimento de grãos [6].

\section{CONCLUSÕES}

Por meio do processo de sinterização por microondas pôde ser observada a densificação das cerâmicas de PZT com um tempo de patamar muito reduzido. $\mathrm{O}$ maior valor de densificação foi obtido para amostra sinterizada a $1100{ }^{\circ} \mathrm{C}$ durante $10 \mathrm{~min}$, o que reduziu a perda de $\mathrm{PbO}$ devido às altas temperaturas de processamento em relação ao convencional, em forno elétrico resistivo. Para esta condição, a cerâmica apresentou um valor de constante dielétrica superior $(\varepsilon=2426)$ aos apresentados para cerâmicas obtidas pelo processo convencional de sinterização. Desta forma, pode-se afirmar que este tipo de processamento, além de viável, se torna bastante interessante no processamento deste tipo de material. 


\section{REFERÊNCIAS}

[1] S. M. Landin, W. A. Schulze, J. Am. Ceram. Soc. 73, 4 (1990) 913.

[2] Z. Xie , Z. Gui , L. Li , T. Su , Y. Huang, Mater. Lett. 36 (1998) 191.

[3] M. V. Gelfuso, Tese de Doutorado, DEMa-UFSCar, S. Carlos, SP (1998).

[4] S. Chiang, M. Nishioka, R. M. Fulrath, J. A. Pask, Am. Ceram. Soc. Bull. 60 (1981) 484.

[5] C. C. Hsueh, M. L. Mecartney, W. B. Harrison, M. R. B. Hanson, B. G. Koepke, J. Mater. Sci. Lett. 8 (1989) 1209.

[6] C. E. Baumgartner, J. Am. Ceram. Soc. 71, 7 (1988) C350.

[7] S. M. Landin, W. A. Schulze, J. Am. Ceram. Soc. 73, 4 (1990) 913.

[8] S. Rhee, T. R. Shrout, G. Link, M. Thumm, J. Am. Ceram. Soc. Japan 111, 5 (2003) 312.
[9] C.-L. Li, C.-C. Chou, J. Phys. IV 128 (2005) 21.

[10] K. Pramod K Sharma, Z. Ounaies, V. V. Varadan, V. K. Varadan, Smart Mater. Struct. 10 (2001) 878.

[11] D. Agrawal , J. Mater. Educ. 19 (1997) 49.

[12] J. Cheng, D. Agrawal, Y. Zhang, R. Roy, Mater. Lett. 56 (2002) 587.

[13] K. H. Brosnan, G. L. Messing, D. K. Agrawal, J. Am. Ceram. Soc. 86 (2003) 307.

[14] L. Wu, C.-C. Lee, T.-S. Wu, C.-C. Wei, Ferroelectrics 41 (1982) 157.

[15] Z. Xie, J. Yang, X. Huang, Y. Huang, J. Eur. Ceram. Soc. 19, 3 (1999) 381.

[16] S. S. Chandratreya, R. M. Fulrath, J. A. Paskreaction, J. Am. Ceram. Soc. 64, 7 (1981) 422.

[17] M. A. Janney, H. D. Kimrey, M. A. Schmidt, J. O. Kiggans, J. Am. Ceram. Soc. 74, 7 (1991) 1675.

(Rec. 28/02/2006, Rev. 08/09/2006, 19/01/2007, Ac. 13/07/2007) 